\title{
2 Wie Klima funktioniert und warum sich die Atmosphäre erwärmt
}

\author{
Volker Wittpahl
}

Klima ist im engeren Sinn definiert als das durchschnittliche Wetter bzw. als die statistische Beschreibung relevanter Größen mittels der Ermittlung von Durchschnitt und Variabilität über Zeitspannen im Bereich von Monaten, Jahren oder gar von Millionen von Jahren. Die Weltorganisation für Meteorologie $(\mathrm{WMO})^{10}$ definiert den Zeitraum zur Mittelung einer Variable mit 30 Jahren; die wesentlichen Variablen sind Temperatur, Niederschlag und Wind. In einem weiter gefassten Sinn ist Klima der Zustand des Klimasystems einschließlich einer statistischen Beschreibung wie zum Beispiel mittlere Jahrestemperatur und -niederschlag aber auch die Eintrittswahrscheinlichkeit bzw. Häufigkeit von Ereignissen wie die mittlere Dauer von Dürren, Sturmhäufigkeit und Starkniederschlägen.

Das Klimasystem besteht im Wesentlichen aus den folgenden Komponenten: Der Atmosphäre, den Ozeanen mit ihrem Meereis und ihrer Biosphäre, der Landoberfläche mit der Biosphäre an Land sowie den unterirdischen Wasserflüssen und den Eisschilden inklusive dem Schelfeis (Brasseur et al. 2017:8). Veränderungen im Klima können sich durch interne Wechselwirkungen im Klimasystem ergeben oder durch externe Einflüsse, zu denen unter anderem Änderungen der Sonneneinstrahlung, Schwankungen der Erdbahnparameter und Vulkanausbrüche gehören. Hinzu kommen noch die anthropogenen, also die durch Menschen verursachten Einflüsse wie die Änderung der Konzentrationen von Spurengasen und Aerosolen in der Atmosphäre und die Landnutzung.

\section{Aufbau der Erdatmosphäre}

Die Atmosphäre ist von der Erdoberfläche aus betrachtet in fünf aufeinander folgende Bereiche unterschiedlicher Ausdehnung unterteilt, die in Abb. 2.1 logarithmisch dargestellt sind. In ihren Übergangsgebieten ist die Temperatur in Bereichen von jeweils rund 10 Höhenkilometern ungefähr konstant. Diese Übergangsbereiche tragen in ihrer Bezeichnung die Endung "Pause", sodass es die Tropopause, Stratopause und Mesopause gibt. Für die meisten Betrachtungen sind aber nur die beiden erdnächsten Schichten Troposphäre und Stratosphäre relevant.

10 Englisch: World Meteorological Organization (WMO). 


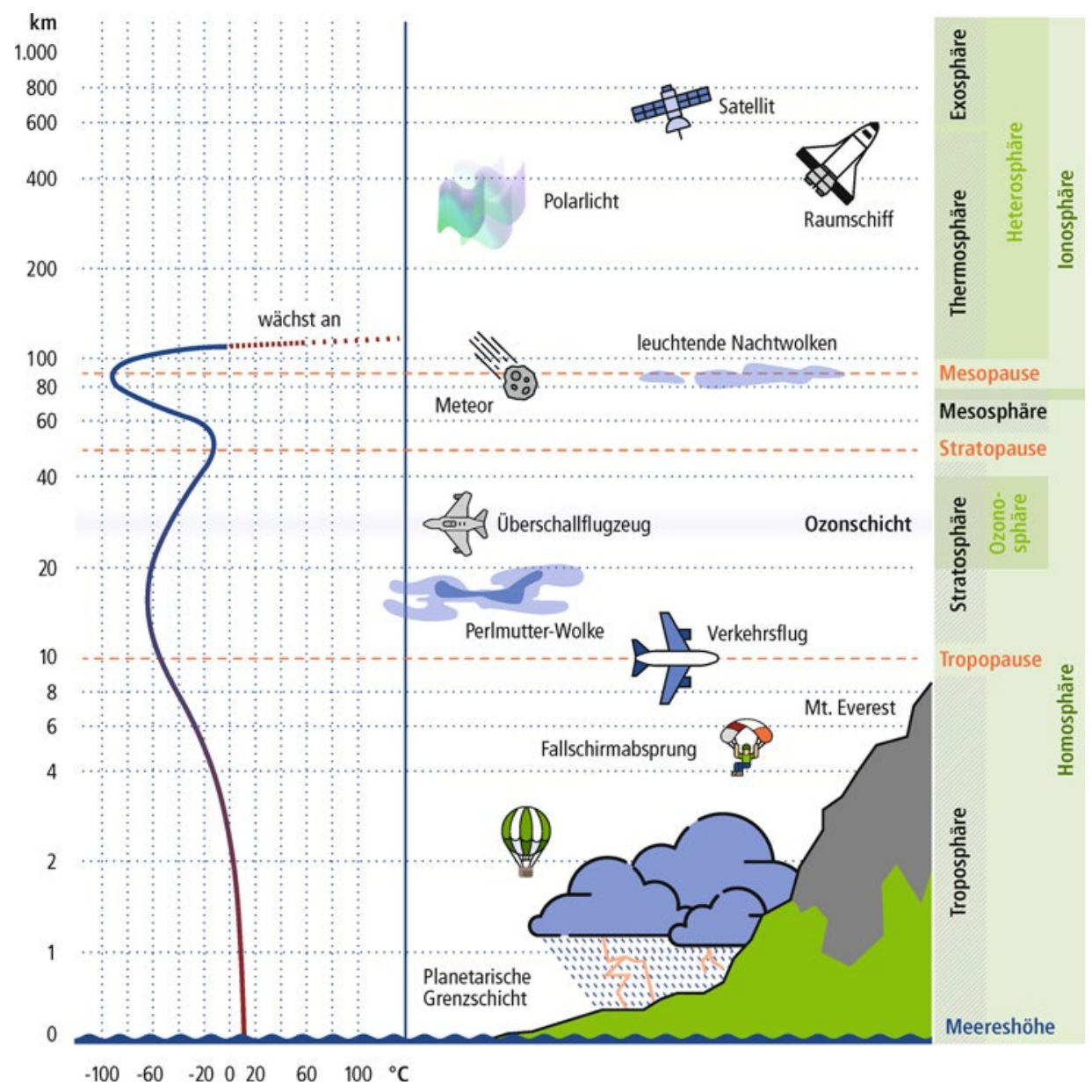

Abb. 2.1 Aufbau der Atmosphäre und Temperaturverlauf in logarithmischer Darstellung entlang der Höhe über dem Meeresspiegel. (Eigene Darstellung nach Klose 2016:16)

Für diese beiden Schichten gibt es Höhenabhängigkeiten für den Luftdruck, die Teilchenzahldichte und die Temperatur. Man sieht in Abb. 2.1, wie die Temperatur in den ersten Höhenkilometern linear abfällt und in der Tropopause ihren Tiefstwert von etwa 217 Kelvin (-56 Grad Celsius) erreicht. Verursacht wird diese adiabatische Abkühlung durch die Arbeit, welche das Gas leisten muss, wenn es gegen die Schwerkraft in die Höhe aufsteigt. Diese Energie entzieht sich der Bewegungsenergie der Gas-Teilchen, was sich als Temperaturabsenkung bemerkbar macht. Durch die Strahlungsheizung der Sonnenstrahlung steigt die Temperatur in der Stratosphäre 
wieder an, bis sie in der Stratopause ein relatives Temperaturmaximum von 271 Kelvin (-2 Grad Celsius) erreicht.

Während sich schwere Teilchen in der Regel am Boden ansammeln, wie zum Beispiel in Sedimenten, sorgt in der Atmosphäre der Effekt des turbulenten Transports für eine starke Durchmischung der Teilchen bis hinauf zu einer Höhe von circa 70 Kilometer über dem Meeresspiegel. Diese Durchmischung bedingt auch, dass die Teilchen in der Troposphäre eine mittlere Verweildauer von einigen Wochen haben.

In Abb. 2.2 sind die wichtigsten Bestandteile der Atmosphäre aufgeführt. Die Atmosphäre besteht zu 99 Prozent aus Stickstoff $\left(\mathrm{N}_{2}\right)$ und Sauerstoff $\left(\mathrm{O}_{2}\right)$ sowie zu 0,9 Prozent aus dem Edelgas Argon (Ar). Die restlichen 0,04 Prozent der Atmosphäre machen Spurengase aus. Das Spurengas Kohlendioxid $\left(\mathrm{CO}_{2}\right)$ hat inzwischen eine Konzentration von circa 410 ppm ${ }^{11}$ erreicht und stellt den weitaus größten Anteil der Spurengase in der Atmosphäre (Umweltbundesamt 2020). Auch Wasserdampf $\left(\mathrm{H}_{2} \mathrm{O}\right)$ ist ein relevantes Spurengas. Das $\mathrm{H}_{2} \mathrm{O}$ steht dabei weniger für das in den Wolken kondensierende Wasser, sondern vielmehr für freie $\mathrm{H}_{2} \mathrm{O}$-Moleküle in der Atmosphäre. Im Gegensatz zu anderen Spurengasen weist Wasserdampf starke Konzentrationsschwankungen auf. In der oberen Troposphäre und der Stratosphäre finden sich typische Konzentrationen um die 3 ppm, während sich in der unteren Troposphäre Gebiete finden lassen, in denen die Konzentration auf bis zu 3 Prozent ansteigen kann.

Etwa 75 Prozent der Luftmasse ist in der Troposphäre enthalten, die im Verhältnis zum Erddurchmesser von 12.700 Kilometer und mit ihrer Höhe von rund 10 Kilometern nur eine sehr dünne Schicht auf dem Planeten Erde bildet.

\section{Die Energiebilanz in der Erdatmosphäre}

\section{Treibhauseffekt}

Der allgemein verwendete Begriff "Treibhauseffekt" beschreibt die Vorgänge in der Erdatmosphäre, die zu einer Erwärmung der Atmosphäre führen, ist aber eher missverständlich. Die physikalischen Zusammenhänge verdeutlicht eine einfache Modellbetrachtung. Zum Verständnis des Einflusses von Einstrahlung und Abstrahlung auf die Atmosphäre wird zunächst ein kugelförmiger Himmelskörper ohne Lufthülle angenommen. Die Einstrahlung von der Sonne beträgt 1,368 kW/ $\mathrm{m}^{2}$. Die sonnenabgewandte Halbkugel des Himmelskörpers liegt im Schatten und wird nicht bestrahlt, während auf der beleuchteten Halbkugel zu ihren Polen die Einstrahlung in einem

11 Teile pro Million Teilchen (Englisch: parts per million; ppm). 


\begin{tabular}{|c|c|c|c|}
\hline & Gas, chem. Formel & Volumenanteil (2016) & Volumenanteil (2018) \\
\hline \multirow{3}{*}{$99,96 \%$} & Stickstoff, $\mathrm{N}_{2}$ & $78,08 \%$ & \\
\hline & Sauerstoff, $\mathrm{O}_{2}$ & $20,95 \%$ & \\
\hline & Argon, Ar & 0,93 \% & \\
\hline \multirow{15}{*}{$0,04 \%$} & Kohlendioxid, $\mathrm{CO}_{2}$ & 0,036 \% (386 ppm) & $407,8 \pm 0,1 \mathrm{ppm}$ \\
\hline & Neon, Ne & $18,2 \mathrm{ppm}$ & \\
\hline & Helium, He & $5,2 \mathrm{ppm}$ & \\
\hline & Methan, $\mathrm{CH}_{4}$ & $1,8 \mathrm{ppm}$ & $1,869 \pm 000,2 \mathrm{ppm}$ \\
\hline & Krypton, Kr & 1,1 ppm & \\
\hline & Wasserstoff, $\mathrm{H}_{2}$ & $0,5 \mathrm{ppm}$ & \\
\hline & Lachgas, $\mathrm{N}_{2} \mathrm{O}$ & $0,3 \mathrm{ppm}$ & $331,1 \pm 0,1 \mathrm{ppb}$ \\
\hline & Xenon, Xe & 0,09 ppm (90 ppb) & \\
\hline & Ozon, $\mathrm{O}_{3}$ & $15-50 \mathrm{ppb}$ & \\
\hline & Stickoxide, $\mathrm{NO}_{x}$ & $0,5-5 \mathrm{ppb}$ & \\
\hline & Schwefeldioxid, $\mathrm{SO}_{2}$ & $0,2-4 \mathrm{ppb}$ & \\
\hline & Ammoniak, $\mathrm{NH}_{3}$ & $0,1-5 \mathrm{ppb}$ & \\
\hline & $\mathrm{FCKW}-12, \mathrm{CF}_{2} \mathrm{Cl}_{2}$ & $\sim 0,5 \mathrm{ppb}$ & \\
\hline & $\mathrm{FCKW}-11, \mathrm{CFCl}_{3}$ & $\sim 0,3 \mathrm{ppb}$ & \\
\hline & FCKW-22, $\mathrm{CHCIF}_{2}$ & $\sim 0,1 \mathrm{ppb}$ & \\
\hline
\end{tabular}

Abb. 2.2 Zusammensetzung der Atmosphäre. Die Konzentration ist angegeben in Prozent sowie ppm (Englisch: parts per million $=10^{-6}$ ) und ppb (Englisch: parts per billion =10-9), die Werte für das Jahr 2016 wurden entnommen aus (Klose 2016:17/18), die für das Jahr 2018 gemessenen Werte stammen aus (WMO 2019).

flacher werdenden Winkel erfolgt, sodass je Quadratmeter Oberfläche immer weniger Energie auftrifft.

Um die Energie auf der Oberfläche des Himmelskörpers zu bestimmen, berechnet man die aufgenommene Gesamtenergie über die Querschnittsfläche Q. Die Fläche ist genauso groß wie der Schatten des Himmelskörpers: $Q=\pi \cdot r^{2}$, wobei $r$ für den Radius des Himmelskörpers steht. Das Verhältnis von $Q$ zur Oberfläche $A$ des Himmelskörpers $\left(A=4 \cdot \pi \cdot r^{2}\right)$ beträgt ein Viertel. Damit ergibt sich für die mittlere Sonneneinstrahlung $S_{0}$ :

$$
S_{0}=1,368 \cdot 0,25 \mathrm{~kW} / \mathrm{m}^{2}=342 \mathrm{~W} / \mathrm{m}^{2}
$$


Körper strahlen Energie in Form von Wärme ab. Abhängig von der Oberflächentemperatur eines Körpers ergeben sich unterschiedliche Abstrahlspektren. Daher muss die kurzwellige Einstrahlung der Sonne als langwellige Wärmestrahlung, das heißt Infrarot-Strahlung (IR), in den Weltraum zurückgestrahlt werden. Für die Abstrahlung I gilt das Stefan-Boltzmann-Gesetz für einen schwarzen Körper:

$$
I=\varepsilon \cdot \sigma \cdot T^{4}
$$

mit der Stefan-Boltzmann-Konstante $\sigma=5,67 \cdot 10^{-8} \mathrm{Wm}^{-2} \mathrm{~K}^{-4}$ und der Emissivität $\varepsilon$, die für eine Abschätzung mit $\varepsilon=1$ angesetzt wird.

Um nun die mittlere Einstrahlung /s an der Oberfläche des Himmelskörpers durch die Sonne zu ermitteln, muss der Anteil des reflektierten Sonnenlichts von der mittleren Sonneneinstrahlung $S_{0}$ abgezogen werden. Über die Albedo ${ }^{12}$ mit $\alpha$ für Felsgestein von 30 Prozent, was der Albedo der Erde entspricht, lässt sich die aufgenommene Leistung bestimmen.

Es ergibt sich für die mittlere Einstrahlung /s:

$$
I_{S}=(1-\alpha) \cdot S_{0}=(1-\alpha) \cdot 342 \mathrm{~W} / \mathrm{m}^{2}=239 \mathrm{~W} / \mathrm{m}^{2}
$$

Die mittlere Abstrahlung $I_{A}$ ist gleich groß:

$$
I_{\mathrm{S}}=I_{\mathrm{A}}=\varepsilon \cdot \sigma \cdot T^{4}
$$

Wenn man diese Gleichung nach $T$ auflöst und $\varepsilon=1$ setzt, erhält man:

$$
T=\sqrt[4]{\frac{(1-\alpha) \cdot I_{S}}{\varepsilon \cdot \sigma}}=\sqrt[4]{\frac{239}{5,7 \cdot 10^{-8}}}=255 \mathrm{~K}
$$

Ob eine Atmosphäre vorhanden ist oder nicht, spielt keine Rolle, solange die Atmosphäre für die Strahlung "durchsichtig" ist und keine Strahlung in ihr absorbiert wird. Die drei Hauptgase Stickstoff $\left(\mathrm{N}_{2}\right)$, Sauerstoff $\left(\mathrm{O}_{2}\right)$ und Argon (Ar) machen 99,96 Prozent der Erdatmosphäre aus, absorbieren aber kein Infrarotlicht. Hierdurch ist es möglich, dass die Erde den Großteil der von der Sonne aufgenommenen Strahlung wieder in den Weltraum abstrahlt. Dafür absorbieren aber alle Spurengase infrarote Strahlung bei unterschiedlichen diskreten Wellenlängen und sind trotz ihrer sehr geringen Konzentration von großer Bedeutung für das Klima.

12 Albedo beschreibt das Maß für die Reflexionsstrahlung, das heißt für das Rückstrahlvermögen nicht selbst leuchtender, diffus reflektierender Oberflächen und wird oft in Prozent angegeben. So ist die Albedo für schneebedeckte Oberflächen höher als für Böden oder Ozeane. Die Albedo variiert auf der Erde wegen Änderungen der Bodenbedeckung und unterschiedlicher Bewölkung, Schnee-, Eis- und Laubbedeckungen. 

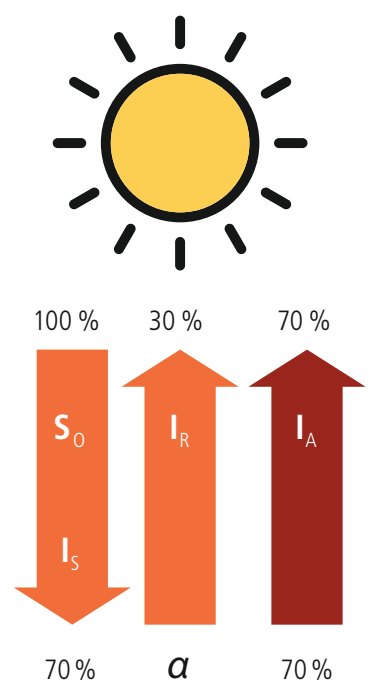
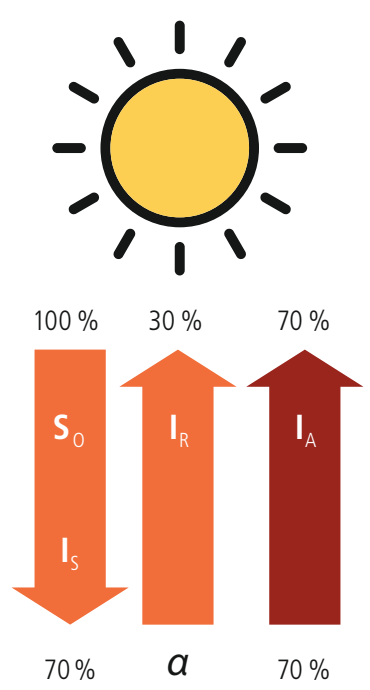

Himmelskörper

ohne Atmosphäre

$\mathrm{T}=255 \mathrm{~K}\left(-18^{\circ} \mathrm{C}\right)$
Atmosphäre

Erde mit

Atmosphäre aus

$\mathrm{N}_{2}, \mathrm{O}_{2}$ und $\mathrm{Ar}$

$\mathrm{T}=255 \mathrm{~K}\left(-18^{\circ} \mathrm{C}\right.$

Abb. 2.3 Einfaches Modell für die Strahlungsbilanz. Links ist das Modell für einen Himmelskörper ohne Atmosphäre gegeben. Rechts ist das Modell für eine Erde mit einer Atmosphäre aus $\mathrm{N}_{2}, \mathrm{O}_{2}$ und Ar gegeben. Die orangen Pfeile repräsentieren die kurzwellige Sonnenstrahlung, die roten Pfeile langwellige Wärmestrahlung.

Wie Abb. 2.3 zeigt, würde die Erde demnach auch eine mittlere Oberflächentemperatur von 255 Kelvin (-18 Grad Celsius) besitzen, wenn ihre Atmosphäre nur aus $\mathrm{N}_{2}, \mathrm{O}_{2}$ und $\mathrm{Ar}$ bestehen würde und keinerlei Spurengase besäße oder sie komplett atmosphärenlos wäre. In beiden Fällen wäre die Erde allerdings ein Eisplanet.

Um den Einfluss der Spurengase in die Modellbildung der Energiebilanz einzubauen, lässt sich die Beschreibung der Erde hin zu einem kugelförmigen "Treibhaus" erweitern. In der Modellvorstellung wird die Erdatmosphäre dabei um eine Scheibe aus "Spezialglas" ergänzt, die die Erde kugelförmig umschließt. Das "Spezialglas" repräsentiert eine sehr hohe Konzentration von $\mathrm{CO}_{2}, \mathrm{H}_{2} \mathrm{O}$ und weiterer Spurengase. Durch die Eigenschaft des „Spezialglases” lässt die Scheibe das sichtbare, kurzwel- 
lige Sonnenlicht passieren und absorbiert die langwellige, infrarote Wärmeabstrahlung vollständig.

In der Konsequenz wird die Glasscheibe nicht durch die kurzwellige Strahlung des Sonnenlichtes $/ s$, sondern durch die Erdabstrahlung $I_{E}$ erwärmt. Die Scheibe unserer Treibhaus-Kugel erhitzt sich und strahlt ihrerseits Wärme ab. Diese Abstrahlung erfolgt über die gesamte Oberfläche der Scheibe, das heißt über die "Innenseite" als Gegenstrahlung IGE zurück zur Erde und über die "Außenseite" in den Weltraum als $I_{G W}$. Auch ohne Berechnung wird nun klar, dass die Erdoberfläche einen Teil ihrer Abstrahlung lE zurückerhält und sich dadurch erwärmt.

Setzt man die einfallende solare Strahlung So mit $342 \mathrm{~W} / \mathrm{m}^{2}$ als Startwert mit 100 Prozent an, so ergibt sich der direkt diffus reflektierte kurzwellige Anteil, der nicht an der Scheibe absorbiert wird und in den Weltraum zurückgestrahlt, entsprechend als $I_{R}=103 \mathrm{~W} / \mathrm{m}^{2}$ bzW. 30 Prozent.

Folglich ergibt sich als absorbierter Anteil labs für die Erdoberfläche 70 Prozent gemäß:

$$
l_{\mathrm{abs}}=(1-\alpha)=239 \mathrm{~W} / \mathrm{m}^{2}
$$

Zur weiteren Vereinfachung der Betrachtung wird davon ausgegangen, dass sich das System in den Endzustand eingeschwungen hat bzw. im Gleichgewichtszustand befindet. Dies bedeutet, dass die Glasscheibe sich soweit aufgeheizt hat, dass die gesamte Abstrahlbilanz in den Weltraum der Einstrahlung $S_{0}$ durch die Sonne entspricht:

$$
S_{0}=I_{R}+I_{G W}
$$

Daraus ergibt sich, dass die langwellige Abstrahlung /GW entsprechend 70 Prozent von $S_{0}$ ist, was einem Wert $I_{G W}$ von $239 \mathrm{~W} / \mathrm{m}^{2}$ entspricht. Da diese Abstrahlung zu beiden Seiten der Scheibe, also Richtung Erdoberfläche $\left(I_{\mathrm{GE}}\right)$ und Richtung Weltall $\left(I_{\mathrm{GW}}\right)$, gleich groß ist, ergibt sich für die Abstrahlung der gesamten Scheibenoberfläche:

$$
I_{\mathrm{GW}}+I_{\mathrm{GE}}=2 \cdot I_{\mathrm{GW}}=478 \mathrm{~W} / \mathrm{m}^{2}
$$

Zum Ausgleich der Strahlungsbilanz muss die Abstrahlung von der erwärmten Erdoberfläche ausgeglichen werden und beträgt demnach

$$
I_{\mathrm{E}}=478 \mathrm{~W} / \mathrm{m}^{2}
$$

Obwohl dieser Wert doppelt so groß ist wie die direkt von der Oberfläche absorbierte Sonneneinstrahlung von $(1-\alpha) S_{0}=239 \mathrm{~W} / \mathrm{m}^{2}$, lässt sich das Ergebnis widerspruchsfrei erklären. Die Einstrahlung der Sonne treibt einen mächtigen Energiestrom an, der zwischen der Erdoberfläche und der Scheibe die Erde umkreist. In diesem ver- 
einfachten Modell für die Atmosphäre muss er sogar höher sein als die gesamte absorbierte Einstrahlung von $239 \mathrm{~W} / \mathrm{m}^{2}$, da der einzige Verlustmechanismus die Abstrahlung in den Weltraum ist. Solange die Reibungsverluste die Antriebsleistung nicht ausgleichen, wird die kinetische Energie ständig zunehmen. Wie ein atmosphärisches vertikales Zirkulationsrad schaukelt sich diese "Strahlungszirkulation" auf, da die direkte Wärmeabstrahlung von der Erdoberfläche ins All in der sehr vereinfachten Betrachtung vollständig unterbunden wurde. Nur die Glasscheibe wirkt hier als Abstrahlschicht mit einer charakteristischen Temperatur $T_{\mathrm{s}}$. An der Scheibe gibt es jedoch zwei Oberflächen, die nach dem Stefan-Boltzmann-Gesetz abstrahlen, wobei nur eine ins All abstrahlen kann und die andere zur Erde gewandt ist. Da die Abstrahlung ins Weltall $I_{\mathrm{GW}}=239 \mathrm{~W} / \mathrm{m}^{2}$ mit der vierten Potenz einhergeht, ergibt sich für die Temperatur $T_{\mathrm{s}}$ der Glasscheibe:

$$
T_{\mathrm{S}}=\sqrt[4]{\frac{239}{5,7 \cdot 10^{-8}}}=255 \mathrm{~K}
$$

Damit entspricht die Temperatur $T_{\mathrm{s}}$ der Glasscheibe der Temperatur der atmosphärenlosen Erde von 255 Kelvin (-18 Grad Celsius), während sich für die Erdoberfläche durch die Abstrahlung von $478 \mathrm{~W} / \mathrm{m}^{2}$ eine um $\sqrt[4]{2}=1,189$ höhere Temperatur $T_{\mathrm{E}}$ mit dem Wert von 303 Kelvin (30 Grad Celsius) ergibt. Durch die Glasplatte wird in dem Modell eine so kräftige Rückstrahlung erzeugt, dass die Oberflächentemperatur $T_{E}$ um 48 Grad Celsius ansteigt. Das Modell ist in der Mitte von Abb. 2.4 gegeben. Seine Vereinfachung repräsentiert die allgemein beschriebene Funktion des Treibhauseffekts. Zusätzlich sind in Abb. 2.4 noch die Strahlungsspektren am Erdboden sowie in der oberen Atmosphäre angegeben, links für die kurz- und rechts die langwellige Strahlung. Die Spektren verdeutlichen den Einfluss der Spurengase.

Der natürliche Treibhauseffekt aufgrund der Konzentration der Spurengase führt zu einer Erwärmung der Erdoberfläche um rund $33 \mathrm{Grad}$ Celsius, das heißt von $255 \mathrm{Kel}-$ vin (-18 Grad Celsius) auf ca. 288 Kelvin (15 Grad Celsius). Das vereinfachte Modell hat somit einen zu großen Treibhauseffekt, was dadurch bedingt ist, dass die durch die Glasscheibe repräsentierte Atmosphäre die Wärmeabgabe der Erdoberfläche vollständig absorbiert und danach zur Hälfte von dort wieder zurückgestrahlt wird.

Um das Modell der Realität anzupassen, müssen noch weitere Faktoren in die Beschreibung eingefügt werden. So ist die Atmosphäre durch die Spurengase für die langwellige Strahlung nicht zu 100 Prozent strahlungsundurchlässig. Auch müsste man mehrere Scheiben im Modell hintereinander anordnen, um das Temperaturprofil der Atmosphäre nachzubilden, und diese noch durch die einfallende Sonneneinstrahlung erwärmen lassen. Zudem sind da noch Wasserdampf und Wolken wie auch Schmutzpartikel in der Luft, die in die Modellbildung einfließen. 


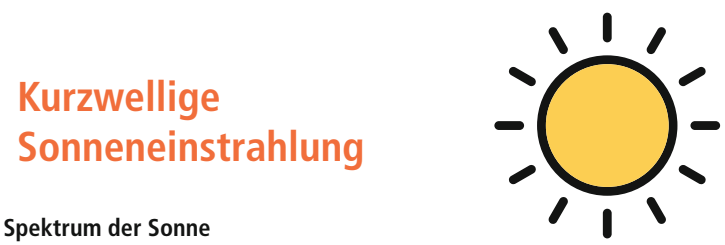

\section{Langwellige Wärmestrahlung}
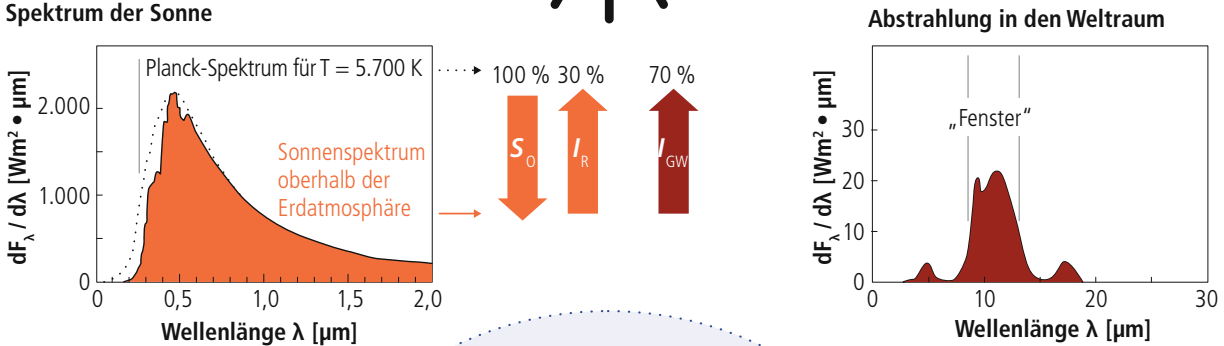

Spektrum auf Meereshöhe nach Absorption durch Wasserdampf und Ozon
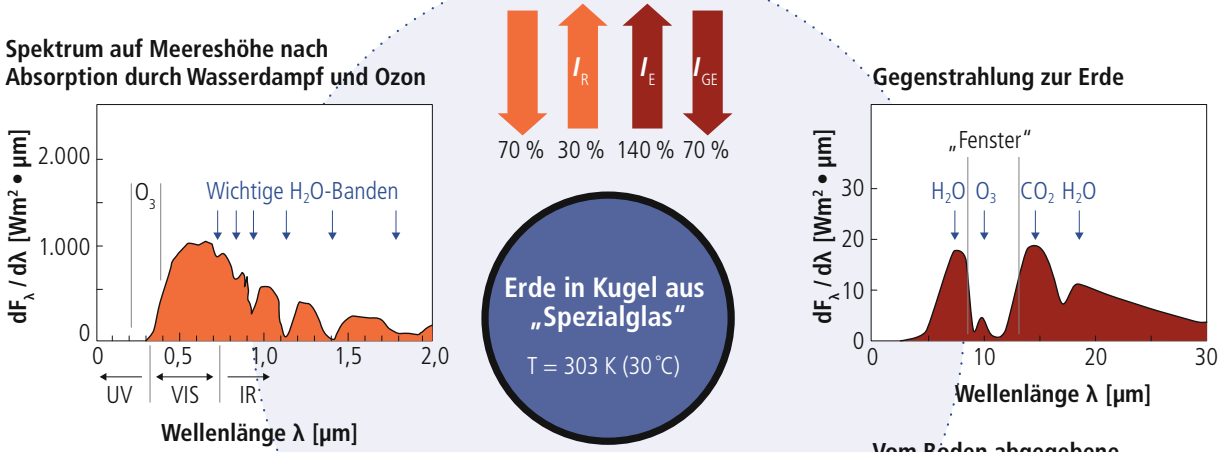

Vom Boden abgegebene Wärmestrahlung

"Scheibe" aus $\mathrm{CO}_{2}, \mathrm{H}_{2} \mathrm{O}$ und weiteren Spurengasen $T=255 \mathrm{~K}\left(-18^{\circ} \mathrm{C}\right)$

Atmosphäre

Weltraum

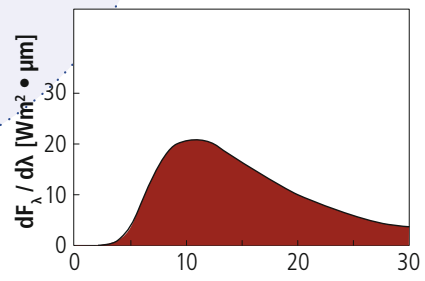

Wellenlänge $\lambda$ [ $\mu \mathrm{m}]$ 
Abb. 2.4 Vereinfachte Beschreibung des Treibhauseffekts und Strahlungsspektren. In der Mitte sieht man das Modell der Treibhaus-Kugel mit den berechneten Austauschströmen. Die Atmosphäre wird dabei begrenzt durch eine Scheibe in Höhe der Stratopause, die für das sichtbare Licht transparent ist und wie eine Glasplatte wirkt, die aber für infrarote Strahlung (IR) intransparent ist und wie eine Wand wirkt. Zusätzlich sind im Bild die Strahlungsspektren am Erdboden und in der Atmosphäre gegeben. Links oben ist vereinfacht das Spektrum des einfallenden Sonnenlichts gegeben. Die Kurve der Spektralverteilung an der AtmosphärenObergrenze zeigt, dass sich die Messung sehr gut mit der Planck-Funktion (gepunktete Kurve) unter der Annahme einer Sonnenoberflächentemperatur von 5700 Kelvin beschreiben lässt. Das Spektrum deckt den Bereich von 0,2 bis $3 \mu \mathrm{m}$ ab und reicht von ultravioletten Bereich (UV) über das sichtbare Spektrum (VIS) bis in den infraroten Bereich (IR). Im Diagramm unten links ist die Spektralverteilung zu sehen, die am Boden ankommt. In ihr ist der UVAnteil für die Wellenlängen $\lambda<0,4 \mu m$ aufgrund der Absorption durch Ozonmoleküle $\left(\mathrm{O}_{3}\right)$ stark reduziert und die Einbrüche im Infrarotbereich $(0,8<\lambda<2 \mu \mathrm{m})$ sind auf die Absorption an Wassermolekülen $\left(\mathrm{H}_{2} \mathrm{O}\right)$ zurückzuführen. Unten rechts in ein Diagramm mit dem vom Boden ausgehenden IR-Spektrum gegeben. Die mittlere Oberflächentemperatur der Erde liegt bei 288 Kelvin und strahlt im Bereich von 3 bis $60 \mu m$ Wellenlänge IR-Wärmestrahlung ab. In dem Diagramm darüber ist das von der Atmosphäre zur Oberfläche zurückgestrahlte IR-Spektrum angegeben, die sogenannte Gegenstrahlung. Das Maximum bei $\lambda=7 \mu \mathrm{m}$ lässt sich auf die Gegenstrahlung durch $\mathrm{H}_{2} \mathrm{O}-$ Moleküle und das Maximum bei $\lambda=13 \mu \mathrm{m}$ auf die Gegenstrahlung von $\mathrm{CO}_{2}$-Molekülen zurückführen. Oberhalb von $\lambda=20 \mu \mathrm{m}$ ist die Gegenstrahlung durch Wassermoleküle auch sehr intensiv. Das Diagramm rechts oben zeigt das IR-Spektrum, das bei wolkenlosem Himmel in den Weltraum abgestrahlt wird. Es stellt die Differenz der Spektren aus den darunter befindlichen Diagrammen dar. Steigt die Konzentration der $\mathrm{CO}_{2}$-Moleküle, so wird der Fensterbereich von der rechten Seite beschnitten und verengt sich, wodurch weniger abgestrahlte Leistung in den Weltraum gelangen kann. Die Verläufe der Spektren in den Diagrammen sind qualitativ dargestellt. (Eigene Darstellung der Spektren nach Roedel 2000)

Wie aus der Modellbetrachtung hervorgeht, ist eine genaue Ermittlung der Energiebilanz für die Atmosphäre von vielen Faktoren abhängig. Hinzu kommt, dass räumliche und zeitliche Schwankungen der Abstrahlung der Erde eine zuverlässige Messung der Strahlungsbilanz äußerst schwierig machen. So sind etwa die Einflüsse von Wolken, Aerosolen und städtischen Gebieten erheblich.

Strahlungsbilanz

Die Berechnung der Energiebilanz über zusätzliche Austauschströme erweitert das Modell der Treibhaus-Kugel und ist in Abb. 2.5 dargestellt. Die Zahlen stehen für globale Mittelwerte der einzelnen Energieflüsse, die eine insgesamt ausgeglichene 
Energiebilanz beschreiben. Auch wenn die Energieflüsse einen quantitativen Einblick in die wesentlichen Komponenten der Energiebilanz der Erde geben, gibt es regional große Unterschiede. Diese stehen in Zusammenhang mit vielen Faktoren, die das Klima der Erde bestimmen, wie die Neigung der Erdachse, die Verteilung der Wasserflächen oder die atmosphärische und ozeanische Zirkulation.

Man sieht, dass etwa 31 Prozent der einfallenden Strahlung $S_{0}$ von Aerosolen in der Atmosphäre, Wassertropfen in den Wolken und der Erdoberfläche direkt ins Weltall reflektiert werden. Die Albedo des Treibhaus-Kugel Modells stellt mit 103 W/ $\mathrm{m}^{2}$ eine gute Näherung dar. Knapp 20 Prozent der Einstrahlung werden direkt von der Atmosphäre absorbiert und erwärmen die Luft. Um die 50 Prozent der Sonneneinstrahlung, $168 \mathrm{~W} / \mathrm{m}^{2}$, erwärmen die Erdoberfläche, was 20 Prozent weniger ist als im Modell der Treibhaus-Kugel. Die Erde gibt ihre Wärme in Form von Wärmeleitung, Konvektion, Verdunstung und langwelliger Abstrahlung in die Atmosphäre ab. Lediglich ein geringer Teil von 8,8 Prozent wird direkt ins Weltall reflektiert. Damit gibt das Modell der Treibhaus-Kugel eine gut funktionierende Vorstellung von den physikalischen Zusammenhängen und verdeutlicht, warum die Zusammensetzung der realen Atmosphäre so entscheiden ist für die Abstrahlung in den Weltraum.

Die Rückstrahlung zur Erdoberfläche weicht gegenüber dem vereinfachten Modell der Treibhaus-Kugel ab. Hier kommt zum Tragen, dass die Glasscheibe nur eine feste Temperatur besitzt während die Lufttemperatur mit zunehmender Höhe stark abnimmt. Die bisherige Modellbetrachtung ging davon aus, dass sich die Energiebilanz zwischen dem eingestrahlten Sonnenlicht und den von der Erde zurückgestrahlten Spektrum vollständig im Gleichgewicht befindet, das heißt die Erde strahlt genauso viel Energie in den Weltraum zurück, wie sie aufgenommen hat. Dies ist jedoch nicht der Fall, wie Satellitenbilder und Klimamodelle zeigen. Dieses Ungleichgewicht wird mit Hilfe des Strahlungsantriebs beschrieben.

\section{Strahlungsantrieb}

Der Strahlungsantrieb bzw. im Englischen Radiative Forcing (RF) gilt als Maßzahl für die Stärke eines natürlichen oder anthropogenen Faktors auf die Veränderung des Strahlungshaushalts der Atmosphäre und wird in W/ $\mathrm{m}^{2}$ angegeben. Er wird berechnet über die Änderung der Nettoeinstrahlung an der Tropopause als Differenz von Einstrahlung von der Sonne abzüglich der Abstrahlung durch die Erde. Der Strahlungsantrieb kann die durch Treiber verursachte Änderung des Energieflusses quantifizieren. Positive RF-Werte stehen für eine Erwärmung der Erde, da mehr Energie in der Atmosphäre verbleibt als eingestrahlt wird, negative RF-Werte führen zu einer Abkühlung der Erdoberfläche. Der RF kann herangezogen werden, um Störungen durch Konzentrationsänderungen von Treibhausgasen zum Beispiel zwischen dem heutigen Zustand und der vorindustriellen Zeit oder einer in der näheren Zukunft erwarteten Konzentration zu ermitteln. 


\section{Kurzwellige \\ Sonneneinstrahlung}

Einfallende

kurzwellige

Sonnenstrahlung

$342 \mathrm{~W} / \mathrm{m}^{2}$

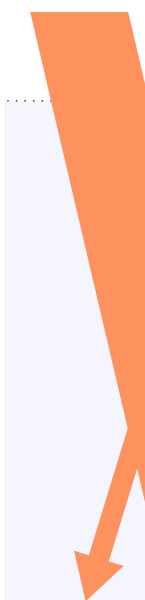

Von der

Atmosphäre

absorbiert

$67 \mathrm{~W} / \mathrm{m}^{2}$

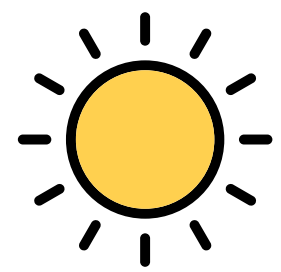

Austretende langwellige

Wärmestrahlung $235 \mathrm{~W} / \mathrm{m}^{2}$

\section{Langwellige \\ Wärmestrahlung}


Schaut man sich die $\mathrm{CO}_{2}$-Konzentration an, so lag sie bis zum Jahr 1800 etwa bei $280 \mathrm{ppm}$ und die Erde befand sich in einem Strahlungsgleichgewicht $\left(l_{\text {ein }}=l_{\text {aus }}\right)$. Eine Störung, zum Beispiel durch eine Verdoppelung der $\mathrm{CO}_{2}$-Konzentration von 280 ppm auf $560 \mathrm{ppm}$ verringert die abgestrahlte Infrarotstrahlung laus, was einer Erhöhung der eingestrahlten Sonnenleistung $\left(\Delta /_{\text {ein }}=R F\right)$ von $R F=3,7 \mathrm{~W} / \mathrm{m}^{2}$ äquivalent ist.

Der Internationale Klimarat (IPCC) bezieht die Änderung des Energieflusses auf das vorindustrielle Jahr 1750. Der globale RF-Wert bezogen auf 1750 beträgt für das Jahr $2019 \mathrm{RF}=3,14 \mathrm{~W} / \mathrm{m}^{2}$ (NOAA 2020). Eine Übersicht über wesentliche Treiber des natürlichen wie auch durch den Menschen verursachten Strahlungsantriebs für exemplarische Jahre gibt Abb. 2.6. Laut IPCC hat der Anstieg der $\mathrm{CO}_{2}$-Konzentration seit 1750 den größten Beitrag zum gesamten Strahlungsantrieb verursacht (IPCC 2013:WGI-11). Man hat festgestellt, dass die tatsächliche Klimaänderung größer ist, als aufgrund des Strahlungsantriebs der Spurengase zu erwarten gewesen wäre. Ursache hierfür sind Rückkopplungseffekte, zum Beispiel durch Wasserdampf in der Atmosphäre oder durch eine Eis-Albedo-Rückkopplung (Brasseur et al. 2017:10).

\section{Der Kohlenstoff-Kreislauf}

Drei Hauptreservoire auf der Erde speichern Kohlendioxid. Neben der Atmosphäre sind dies die Biomasse an Land und die Ozeane. Alle drei Reservoire stehen über Austauschflüsse in einer Wechselwirkung miteinander. Der Austausch zwischen diesen Systemen wird als globaler Kohlenstoff-Kreislauf bezeichnet und ist in Abb. 2.7 in vereinfachter Form dargestellt.

Die $\mathrm{CO}_{2}$-Konzentration in der Atmosphäre wird im Wesentlichen bestimmt durch Austauschflüsse zwischen den Reservoiren. Abb. 2.7 zeigt im oberen Teil den Austausch mit der Atmosphäre und dessen Beeinflussbarkeit. Der Austausch zwischen der Atmosphäre und den Ozeanen findet in großen natürlichen Flüssen statt, die aktuell kaum direkt durch den Menschen beeinflussbar sind. Die $\mathrm{CO}_{2}$-Emissionen aus der Nutzung fossiler Energieträger sind durch den Menschen verursachte Flüsse und somit auch durch den Menschen kontrollierbar. Die $\mathrm{CO}_{2}$-Flüsse zwischen der Biosphäre an Land und der Atmosphäre sind große und überwiegend natürliche Flüsse, welche nur zu einem geringen Teil von Menschen beeinflussbar sind, hierbei maßgeblich durch Landnutzungsänderungen bzw. -management. Die Aufteilung von Zuund Abflüssen zwischen Atmosphäre und Biosphäre an Land lässt sich durch die Ausgestaltung der Landnutzung verschieben, wenn auch in Grenzen. Hierbei ergriffene Maßnahmen sind meist reversibel.

Um den Kohlenstoff-Kreislauf auch quantitativ zu beschreiben, sind Werte aus der Literatur für die Jahre 2006 und 2012 in Abb. 2.7 eingetragen. Hierzu sind die Reser- 


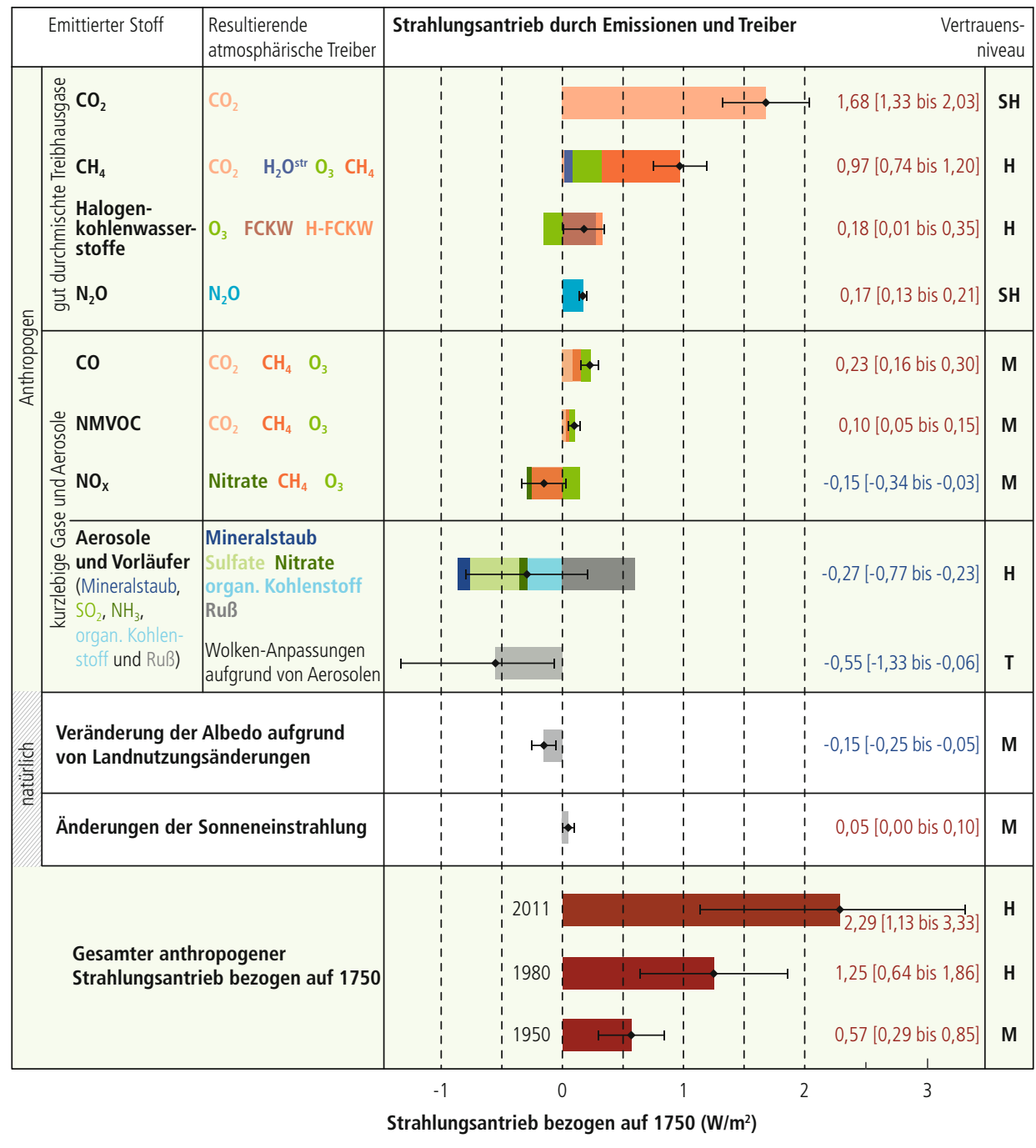

Abb. 2.6 Strahlungsantrieb für unterschiedliche Treiber und Emissionen. Die Abbildung stellt Schätzwerte für das globale Mittel des Strahlungsantriebs (RF) dar. Die Werte sind aus dem Jahr 2011 bezogen auf das Jahr 1750 sowie kumulative Unsicherheiten für die Haupttreiber des Klimawandels. Die schwarzen Rhomben entsprechen den besten Schätzungen mit den entsprechenden Unsicherheitsbereichen. Unten in der Abbildung ist bezogen auf das Jahr 1750 der gesamte anthropogene Strahlungsantrieb für drei exemplarische Jahre gegeben. Rechts findet sich das Vertrauensniveau des Nettoantriebs (SH - sehr hoch, $\mathrm{H}$ - hoch, M - mittel, G - gering, SG - sehr gering). (Eigene Darstellung nach IPCC 2013:WGI-12) 


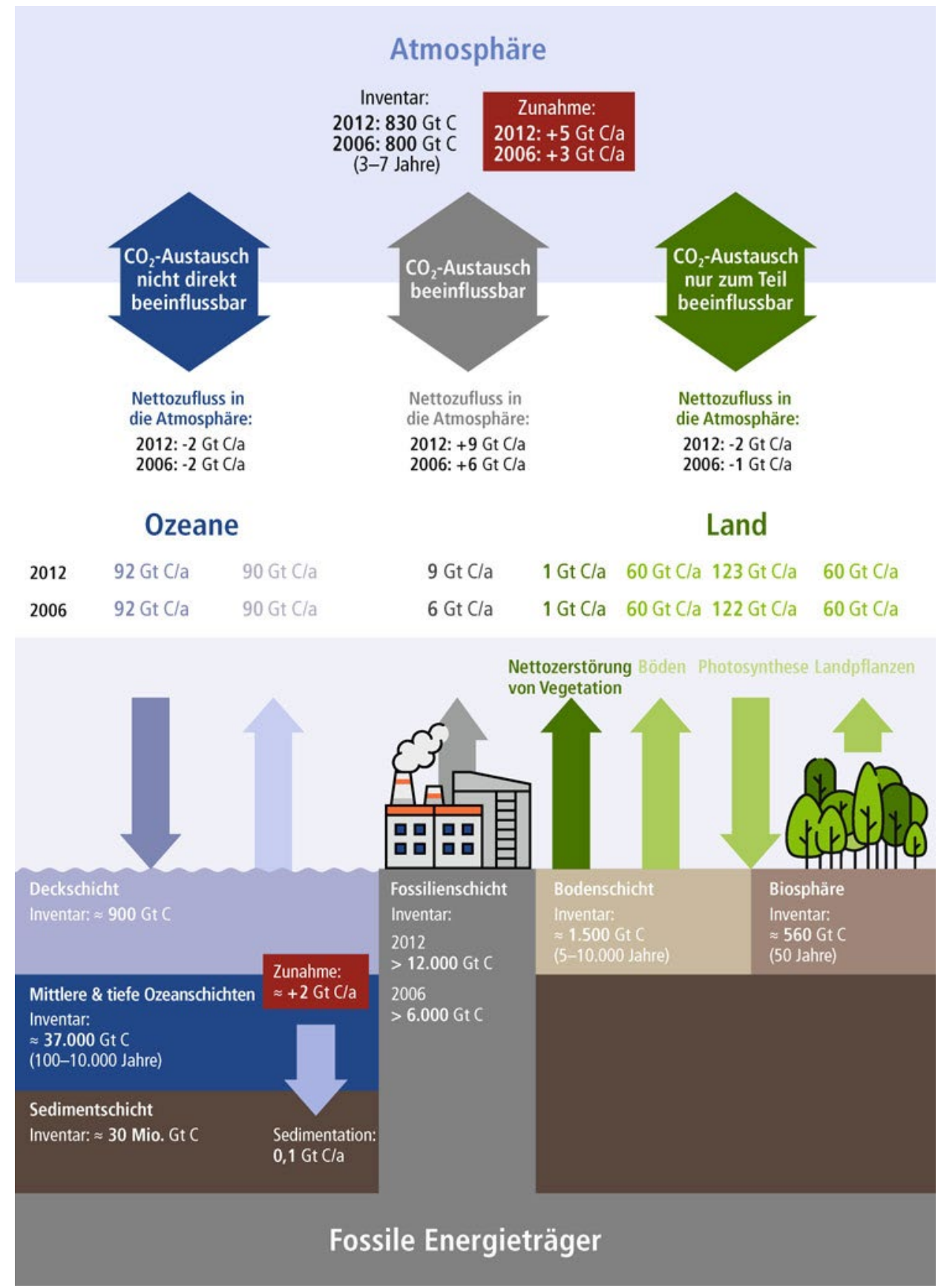


Abb. 2.7 Vereinfachte Darstellung des Kohlenstoff-Kreislaufs. Man sieht in der oberen Bildhälfte die drei Hauptreservoire für Kohlenstoff: Atmosphäre, Ozeane und Land sowie ihre Austauschflüsse inklusive deren Beeinflussbarkeit. In der unteren Bildhälfte sind die Austauschflüsse wie auch die Hauptreservoire weiter unterteilt und mit Werten für die Jahre 2006 und 2012 versehen. Die Werte für die Kohlenstoff-Reservoire sind als Inventare in Gigatonnen Kohlenstoff (Gt C) und die Austauschflüsse in Gt pro Jahr (Gt Cla) angegeben. Die mittlere Verweildauer ist jeweils in Klammern angegeben. Durch die Angaben aus 2006 und 2012 lassen sich die Änderungen im Kohlenstoff-Kreislauf und die Zunahme in der Atmosphäre nachvollziehen. Der Wert von 830 Gt C in der Atmosphäre entspricht einer $\mathrm{CO}_{2}$ Konzentration von 390 ppm. (Eigene Darstellung nach WBGU 2006:67 und WBGU 2013:38)

voir-Kapazitäten als Inventare der verschiedenen Reservoire in Gigatonnen ${ }^{13}$ Kohlenstoff (C) mit fett gedruckten Zahlen angegeben; in runden Klammern die mittlere Verweildauer im Reservoir.

Die Austauschflüsse sind in der unteren Bildhälfte weiter aufgegliedert und geben mit Pfeilen ihre Mengenangaben in Gigatonnen Kohlenstoff pro Jahr (Gt C/a) für die Jahre 2006 und 2012 an. Die sich hieraus ergebenden Nettoströme in die Atmosphäre sind ebenso wie die hieraus resultierende Zunahme in der Atmosphäre in der oberen Bildhälfte ersichtlich.

Zum besseren Modellverständnis werden die Austauschflüsse zwischen Atmosphäre und Land und nochmal in die Reservoire Biosphäre und Bodenschicht unterteilt, um die unterschiedliche Verweildauer des $\mathrm{CO}_{2}$ im Hauptreservoir Land abzubilden. Das Reservoir der Biosphäre umfasst Pflanzen und Wälder, das Reservoir der Bodenschicht Gräser, Moore oder Permafrostböden.

Die Inventare betragen für die Biosphäre 560 Gt und für die Bodenschicht 1500 Gt.

An das Land grenzt nach unten zusätzlich noch die Fossilienschicht, die sich aus den Lagerstätten von Kohle, Öl und Gas zusammensetzt und somit eine reine Teilchenquelle ist, aus der vom Menschen $\mathrm{CO}_{2}$ direkt in die Atmosphäre befördert wird.

131 Gigatonne $=1 \mathrm{Gt}=1$ Milliarde Tonnen $=10^{12} \mathrm{~kg}$. Das ist als Wassermenge ein Würfel mit $1 \mathrm{~km}$ Kantenlänge.

Es sind zwei verschiedene Mengenangaben üblich Gt (C) oder Gt $\left(\mathrm{CO}_{2}\right)$. Da die Molekülmassen für Kohlenstoff $\mathrm{C}=12 \mathrm{~g} / \mathrm{mol}$ und Kohlendioxid $\mathrm{CO}_{2}=44 \mathrm{~g} / \mathrm{mol}$ betragen, gilt, dass $1 \mathrm{Gt}\left(\mathrm{CO}_{2}\right) 12 / 44 \mathrm{Gt}(\mathrm{C})=0,272 \mathrm{Gt}(\mathrm{C})$ enthält. Somit entspricht der Betrag von $1 \mathrm{Gt}(\mathrm{C})$ einem Betrag von 3,667 Gt $\left(\mathrm{CO}_{2}\right)$. 
Das Hauptreservoir der Ozeane lässt sich in eine Deckschicht mit darunterliegender Tiefenschicht unterteilen. Ein Abfluss in die am Meeresgrund befindlichen Sedimentschichten ist eine dauerhafte Möglichkeit, $\mathrm{CO}_{2}$ aus dem Gesamtkreislauf zu entfernen. Die Inventare der Ozeane betragen für die Deckschicht $900 \mathrm{Gt}$, für die ozeanischen Tiefenschichten $37.000 \mathrm{Gt}$ und für die darunterliegenden maritimen Sedimentschichten 30 Millionen $\mathrm{Gt}$ - sie sind das größte $\mathrm{CO}_{2}$-Reservoir der Erde.

Die Bilanz an der Ozeanoberfläche mit einem Abfluss von 90 Gt Kohlenstoff in der Äquatorzone und einem Zufluss von 92 Gigatonnen Kohlenstoff vornehmlich in den äquatorfernen Zonen macht den Ozean zu einer $\mathrm{CO}_{2}$-Senke mit zwei Gt Kohlenstoff pro Jahr. Diese reicht jedoch nicht aus, um den Beitrag der Verbrennung von fossilen Energieträgern durch den Menschen pro Jahr zu kompensieren. Zieht man in die Betrachtung noch die Pflanzen an Land mit ein, so wandeln sie über Photosynthese $\mathrm{CO}_{2}$ in $\mathrm{O}_{2}$ und Glucose, wozu sie aber Wasser und Sonnenlicht benötigen, sodass sie über Nacht kein $\mathrm{O}_{2}$ produzieren, sondern Sauerstoff für die Zellatmung nutzen und $\mathrm{CO}_{2}$ ausstoßen. So erklärt sich der Eintrag von je $60 \mathrm{Gt} C$ pro Jahr in die Atmosphäre und eine Aufnahme von 122 Gt C in 2006 bzw. 123 Gigatonnen C in 2012. Berücksichtigt man den Eintrag von einer Gigatonne $C$ pro Jahr durch Waldbrände und andere Naturzerstörungen, so bleiben eine bzw. zwei Gigatonnen C pro Jahr, die der Atmosphäre durch das Reservoir Land entzogen wurden.

Deutlich wird auch die Veränderung der Inventare und Austauschflüsse zwischen 2006 und 2012, die den kontinuierlichen Anstieg der $\mathrm{CO}_{2}$-Konzentration in der Atmosphäre nachvollziehbar machen. So erhöhte sich in diesem Zeitraum das Inventar für die Atmosphäre von $800 \mathrm{Gt}$ auf $830 \mathrm{Gt}$. Die Menschen bedingte Emission durch Verbrennung fossiler Energieträger stieg von $6 \mathrm{Gt}$ auf $9 \mathrm{Gt}$ an. Davon wurden von den Reservoiren Land und Ozeane in Summe 3 bzw. 4 Gt aufgenommen, sodass der jährliche Nettozufluss in die Atmosphäre von $3 \mathrm{Gt} / a$ auf $5 \mathrm{Gt} / \mathrm{a}$ anstieg.

Wurden im Jahr 2012 noch fünf Gigatonnen Kohlenstoff pro Jahr durch das Handeln der Menschen zusätzlich in die Atmosphäre eingebracht, so werden es im Jahr 2019 zusätzlich etwa sieben Gigatonnen gewesen sein, sofern sich die Austauschflüsse der Atmosphäre mit den Ozeanen und dem Land seither nicht verändert haben.

Auch wenn sich die Kapazitäten der Reservoire nur grob ermitteln lassen, so sind die $\mathrm{CO}_{2}$-Konzentrationen in der Atmosphäre recht genau bekannt. Über die Konzentration der Spurengase in der Vergangenheit geben Messungen an Eisbohrkernen exakt Auskunft. Diese bis zu 3600 Meter langen Eisstangen wurden in Grönland und in der Antarktis aus dem Gletschereis gebohrt. Sie enthalten Gasbläschen, die Auskunft geben über die Zusammensetzung der Erdatmosphäre in unterschiedlichen Epochen der Erdgeschichte. In Abb. 2.8 ist dies anhand von Messungen der Forschungsstation "Wostok" nachzuvollziehen. Man sieht, dass die $\mathrm{CO}_{2}$-Konzentration über den Zeitraum von 400.000 Jahren nie den Wert von 300 ppm überschritten hat. 
Kohlendioxid-Konzentraion [ppm]

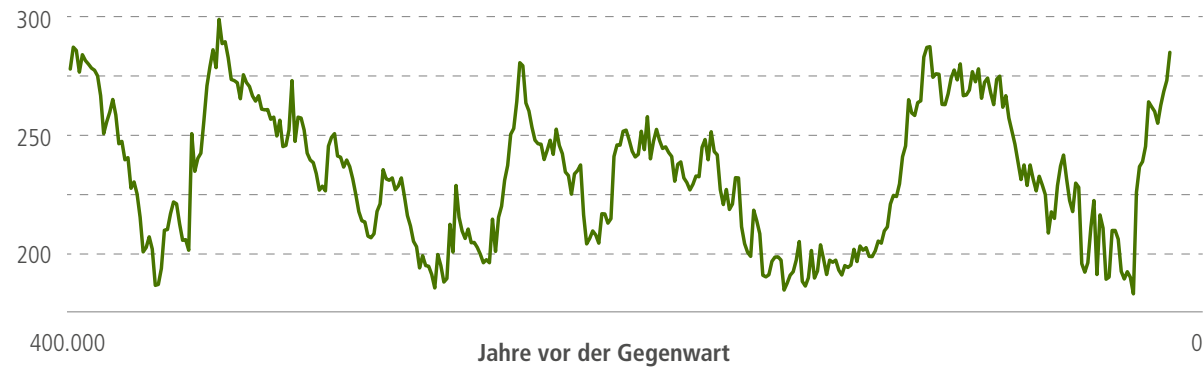

Abb. 2.8 Daten zur $\mathrm{CO}_{2}$-Konzentration in der Atmosphäre über die vergangenen 400.000 Jahre, die anhand eines Eisbohrkerns von der russischen Forschungsstation "Wostok" gewonnen wurden. (Eigene Darstellung nach Barnola 2003)

Ein Beleg für den Einfluss von $\mathrm{CO}_{2}$ auf das Klima ist überdies, dass während der Eiszeiten die $\mathrm{CO}_{2}$-Konzentration auf 180 ppm absank und somit wesentlich niedriger war als in den Warmzeiten.

Seit dem Jahr 1959 wird die $\mathrm{CO}_{2}$-Konzentration in der Atmosphäre systematisch gemessen. Die Monatsmittel (Abb. 2.9) zeigen, dass die Konzentration von 315 ppm im Jahr 1958 auf über 410 ppm angestiegen ist (Umweltbundesamt 2020). Man sieht außerdem im Verlauf der jährlichen Monatsmittel Schwankungen um den Mittelwert, die etwa 3 ppm betragen. Aufgrund der pflanzlichen Photosynthese verringert sich im Sommer auf der nördlichen Halbkugel die $\mathrm{CO}_{2}$-Konzentration um etwa 1,5 ppm, was sich durch die größeren Landmassen der nördlichen Hemisphäre erklären lässt.

\section{Fazit}

Es ist deutlich, dass die Spurengase in der Atmosphäre maßgeblich für das Klima und seine Veränderung verantwortlich sind. Die Energiebilanz der Erdatmosphäre ist derzeit nicht ausgeglichen. Der Strahlungsantrieb RF als Maß für das Ungleichgewicht ist seit Jahren schon positiv, das heißt die Atmosphäre heizt sich weiter auf. Ein maßgeblicher Treiber ist die jährliche Nettozunahme von Kohlendioxid in der Atmosphäre, die in den vergangenen Jahren kontinuierlich gestiegen ist. Die Ursache für den kontinuierlichen Anstieg liegt darin, dass die von den Menschen jährlich verursachten $\mathrm{CO}_{2}$-Emissionen um ein Vielfaches größer sind als die Kapazitäten der natürlichen $\mathrm{CO}_{2}$-Senken an Land und in den Ozeanen.

Was dies bedeutet, macht eine einfache Überschlagsrechnung klar: Angenommen es gelänge, die jährlichen $\mathrm{CO}_{2}$-Emissionen global sofort um 50 Prozent abzusenken. 


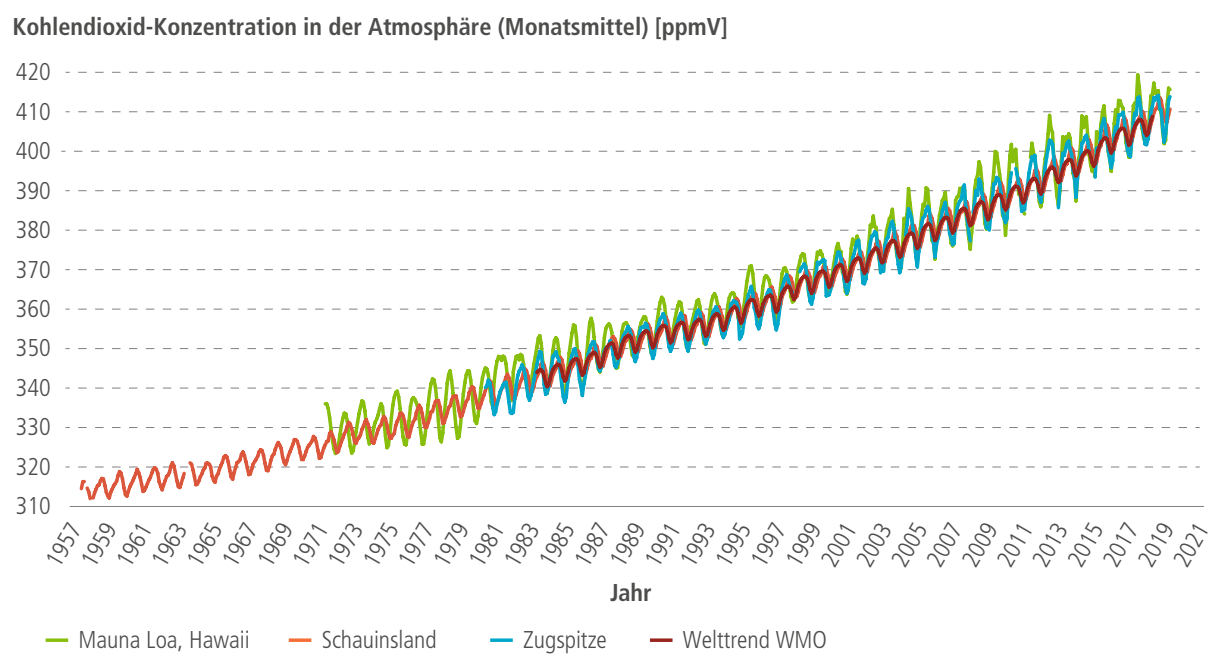

Abb. 2.9 Verlauf der Kohlendioxid-Konzentration für verschiedene Messstationen. Die Kohlendioxid-Konzentration ist angegeben als ppm bezogen auf das Volumen, d. h. 1 ppmV = $10^{-6}=1$ Teil pro Million = 0,0001, angegeben als Molenbruch. (Eigene Darstellung nach Umweltbundesamt 2020)

Auch in diesem Fall würde immer noch eine $\mathrm{CO}_{2}$-Senke in der Größenordnung der gesamten Biosphäre fehlen, um die Nettozunahmen von $\mathrm{CO}_{2}$ in der Atmosphäre auf den Wert Null zurückzuführen. Anders ausgedrückt: Wenn man die jährliche Zunahme der $\mathrm{CO}_{2}$-Konzentration in der Atmosphäre bei gleichbleibender globaler $\mathrm{CO}_{2}$-Emissionen auf den Wert null bringen möchte, so würde man aktuell vier weitere $\mathrm{CO}_{2}$-Senken in der Größenordnung aller auf der Erde vorhandenen Ozeane benötigen.

Bei diesen Dimensionen wird ersichtlich, dass höchstwahrscheinlich auch in 2020erJahren die jährliche Nettozunahme der Atmosphäre mehrere Gigatonnen Kohlenstoff betragen wird - voraussichtlich verbunden mit einer weiteren Beschleunigung der Erderwärmung. 


\section{Literatur}

Barnola, J.M. et al. (2003): Historical CO Record from the Vostok Ice Core, Januar 2003. Verfügbar unter https://cdiac.ess-dive.lbl.gov/ftp/trends/co2/vostok.icecore.co2, zuletzt geprüft am 29.07.2020.

Brasseur, Guy P.; Jacob, Daniela; Schuck-Zöller; Susanne (2017): Klimawandel in Deutschland - Entwicklung, Folgen, Risiken und Perspektiven, Springer Verlag, ISBN 978-3-662-503966. Verfügbar unter https://link.springer.com/book/10.1007\%2F978-3-662-50397-3, zuletzt geprüft am 22.07.2020.

IPCC (2013): KLIMAÄNDERUNG 2013 Naturwissenschaftliche Grundlagen, ISBN 978-3-891 00-048-9, Deutsche Übersetzung durch Deutsche IPCC-Koordinierungsstelle, Bonn, 2016. Verfügbar unter https://www.de-ipcc.de/media/content/AR5-WGI_SPM.pdf, zuletzt geprüft am 02.08.2020.

Klose, Brigitte (2016): Meteorologie: Eine interdisziplinäre Einführung in die Physik der Atmosphäre, 3. Auflage, Springer Verlag, ISBN 9783662436226

NOAA Butler, James H.; Montzka, Stephen A. (2020): THE NOAA ANNUAL GREENHOUSE GAS INDEX (AGGI), Frühjahr 2020 aktualisiert. Verfügbar unter https://www.esrl.noaa. gov/gmd/aggi/aggi.html, zuletzt geprüft am 29.07.2020.

Roedel, Walter (2000): Physik unserer Umwelt: Die Atmosphäre. 3. Auflage, Heidelberg, Springer Verlag, ISBN 3662093251

Umweltbundesamt (2020): Atmosphärische Treibhausgas-Konzentrationen. 24.06.2020. Verfügbar unter https://www.umweltbundesamt.de/daten/klima/atmosphaerische-treibhausgas-konzentrationen, zuletzt geprüft am 29.07.2020.

WBGU (2006): Schubert, Renate; Schellnhuber, Hans Joachim; Buchmann, Nina et al.: Die Zukunft der Meere - zu warm, zu hoch, zu sauer, März 2006, ISBN 3-936191-13-1, S. 67. Verfügbar unter https://www.wbgu.de/fileadmin/user_upload/wbgu/publikationen/archiv/ wbgu_sn2006.pdf, zuletzt geprüft am 29.07.2020.

WBGU (2013): Schubert, Renate; Schellnhuber, Hans Joachim; Buchmann, Nina et al.: Die große Transformation. Klima - kriegen wir die Kurve? Verlagshaus Jacoby\&Stuart, Berlin 2013, ISBN 3-941087-23-1, S. 38.

WMO (2019): WMO GREENHOUSE GAS BULLETIN, Nr. 15, 25. November 2019, ISSN 20780796. Verfügbar unter https://library.wmo.int/doc_num.php?explnum_id=10100, zuletzt geprüft am 26.07.2020.

ZAMG: Zentralanstalt für Meteorologie und Geodynamik (ZAMG), Energiebilanz der Erde. Verfügbar unter https://www.zamg.ac.at/cms/de/klima/informationsportal-klimawandel/ klimasystem/umsetzungen/energiebilanz-der-erde, zuletzt geprüft am 30.07.2020. 


\section{(a) (1)}

Dieses Kapitel wird unter der Creative Commons Namensnennung 4.0 International Lizenz http://creativecommons.org/licenses/by/4.0/deed.de) veröffentlicht, welche die Nutzung, Vervielfältigung, Bearbeitung, Verbreitung und Wiedergabe in jeglichem Medium und Format erlaubt, sofern Sie den/die ursprünglichen Autor(en) und die Quelle ordnungsgemäß nennen, einen Link zur Creative Commons Lizenz beifügen und angeben, ob Änderungen vorgenommen wurden.

Die in diesem Kapitel enthaltenen Bilder und sonstiges Drittmaterial unterliegen ebenfalls der genannten Creative Commons Lizenz, sofern sich aus der Abbildungslegende nichts anderes ergibt. Sofern das betreffende Material nicht unter der genannten Creative Commons Lizenz steht und die betreffende Handlung nicht nach gesetzlichen Vorschriften erlaubt ist, ist für die oben aufgeführten Weiterverwendungen des Materials die Einwilligung des jeweiligen Rechteinhabers einzuholen. 